$$
\begin{aligned}
& \text { استغلال مخلفات الذرة ( الكوالح ) في تصنيع الأكواح الحبيبيـة } \\
& \text { نزار قاسم الدايني }
\end{aligned}
$$

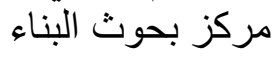

$$
\begin{aligned}
& \text { وز ارة الصناعة و المعادن }
\end{aligned}
$$

\author{
باسم عباس عبد علي مخلي \\ مركز بحوث ومتحف التاريخ الطبيعي \\ جامعة بغداد
}

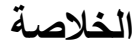

اجري البحث كمحاولة لاستغلال مادة غير مستغلة في العراق وهي كوالح الذرة من خلالال

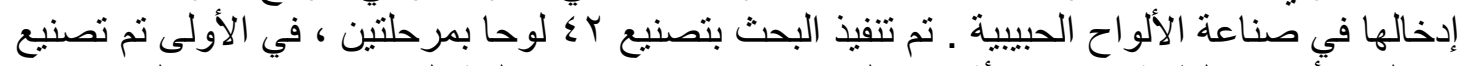

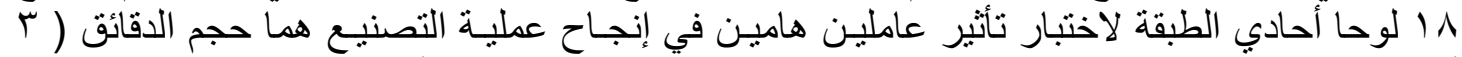

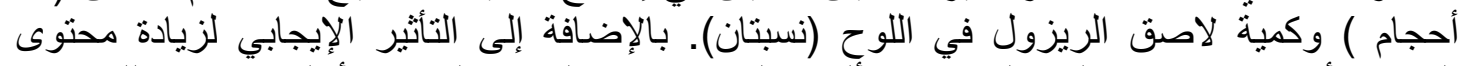

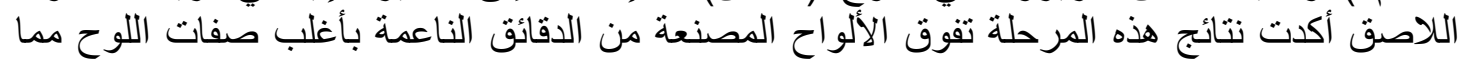

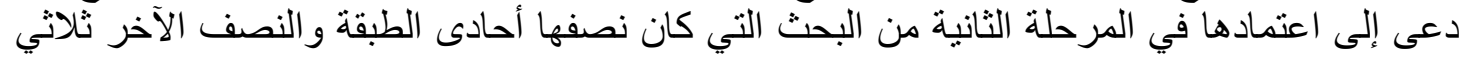

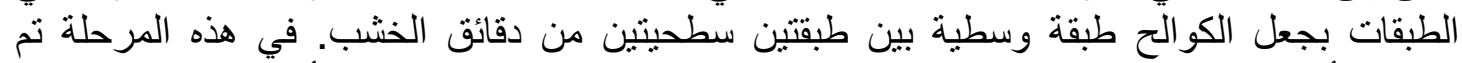

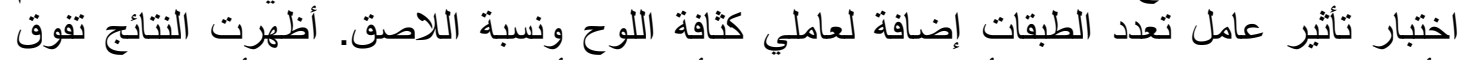

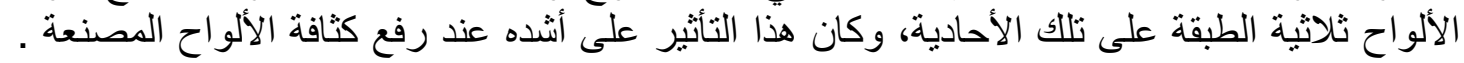

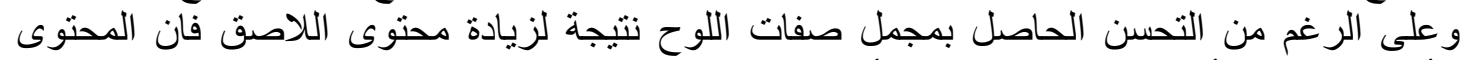

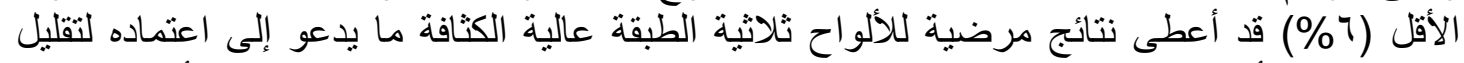

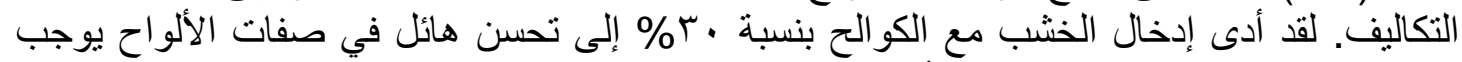
عدم إهماله عند استغلال هذه المادة للألو الح الحبييية .

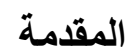

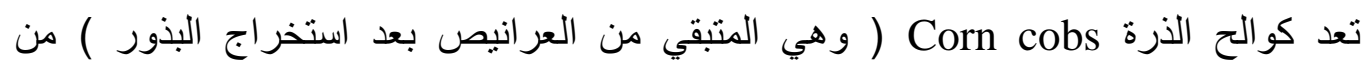

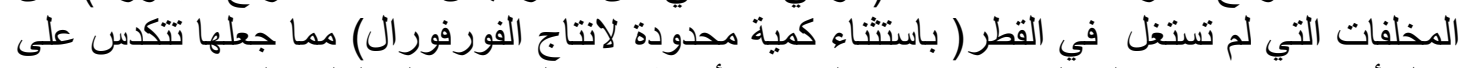

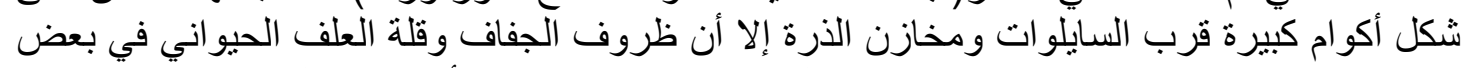

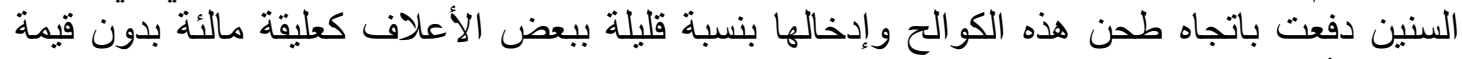

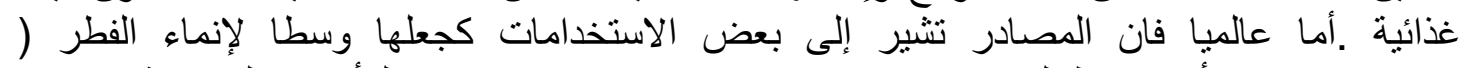
( أو كوسط للإنبات ( Gashroom

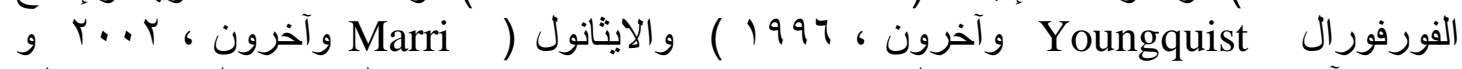

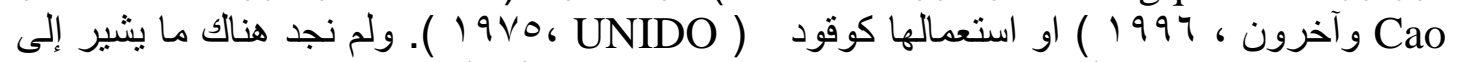

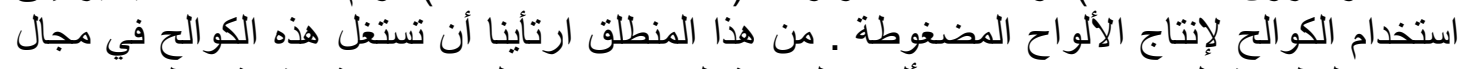

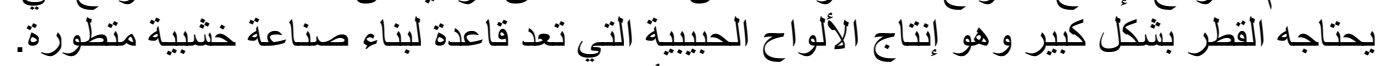

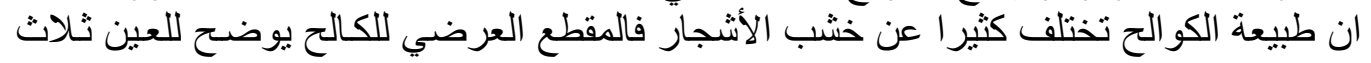

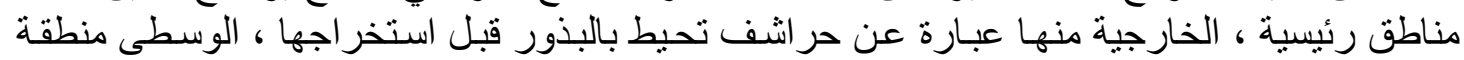

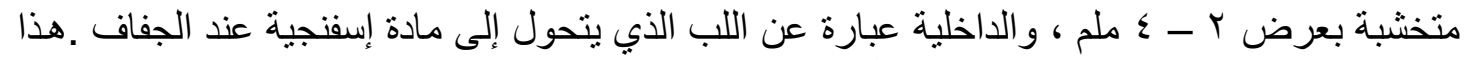

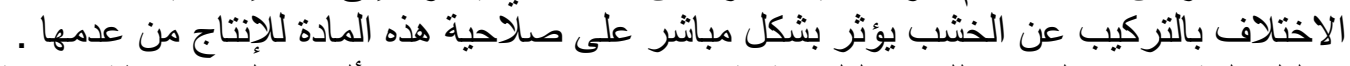

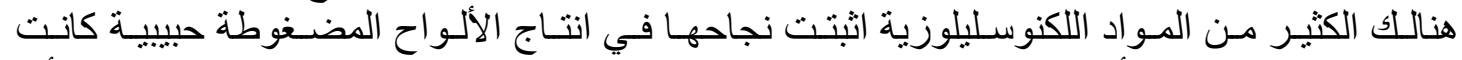

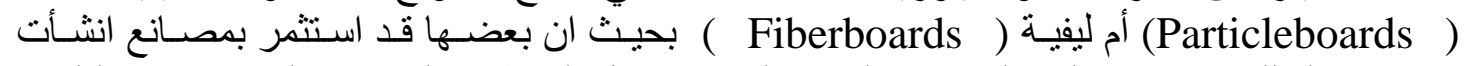

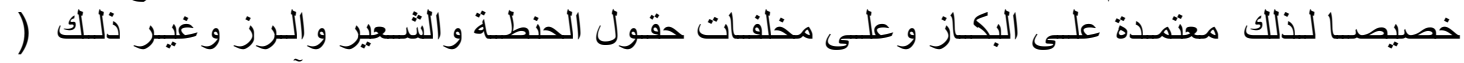

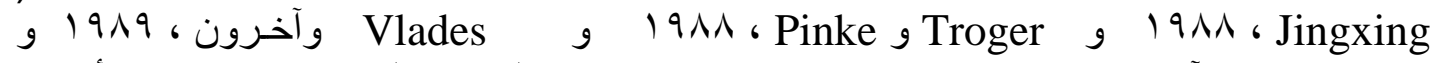

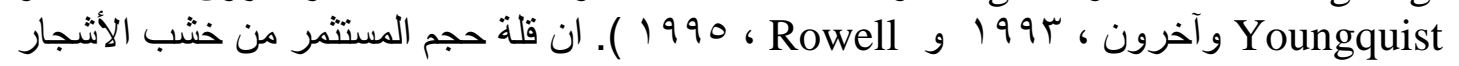

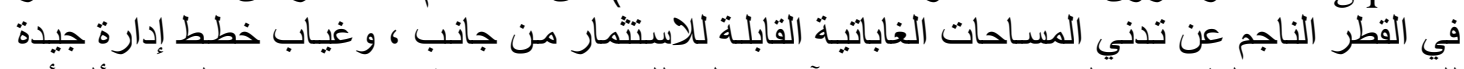

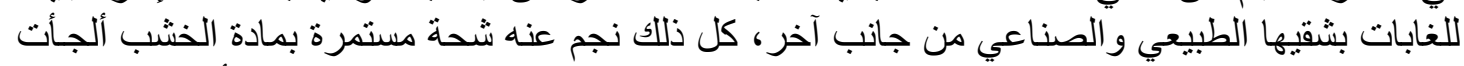

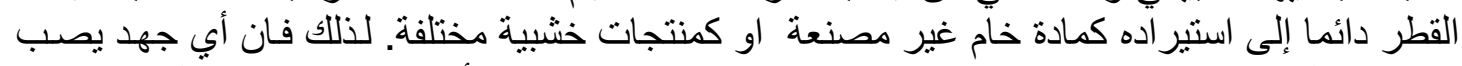

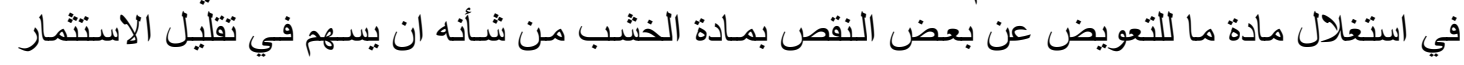




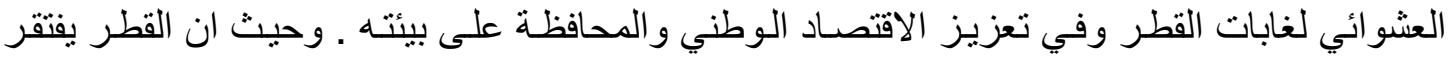

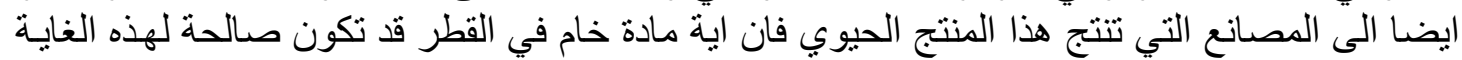

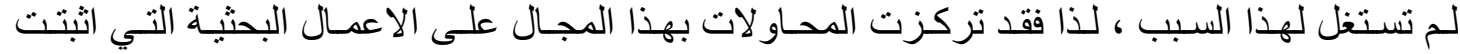

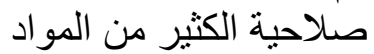

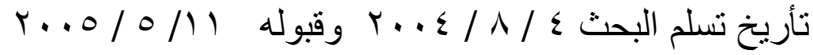

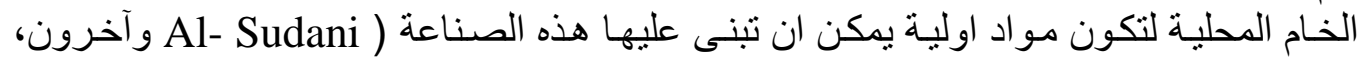

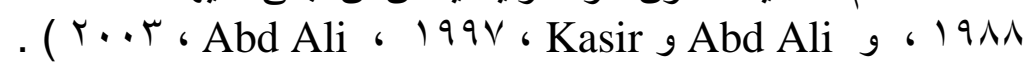

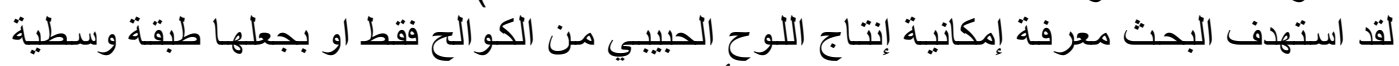

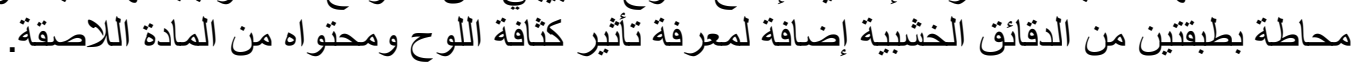

\section{مواد وطرق البحث}

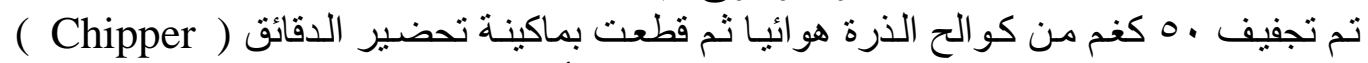

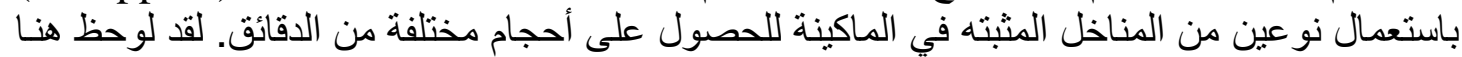

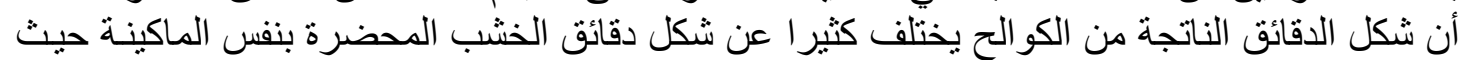

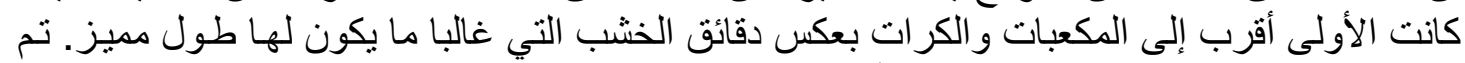
تصنيف الدقائق حجميا بغر ابيل إلي ثلاثثة أحجام : ناعمـة (

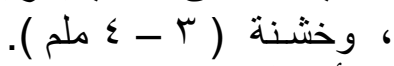

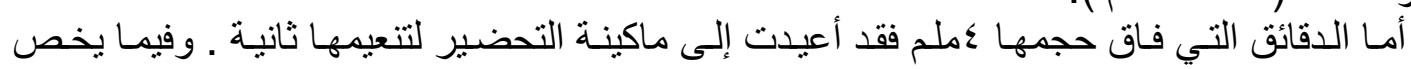

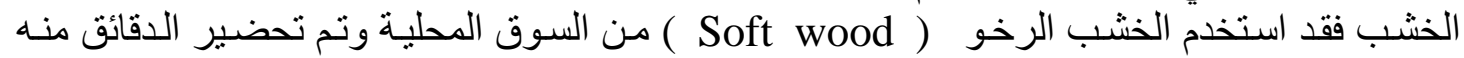

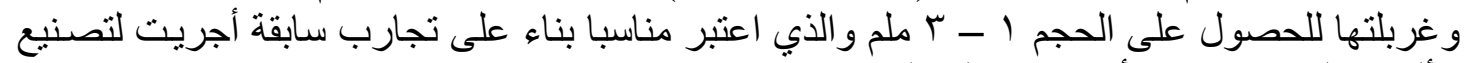

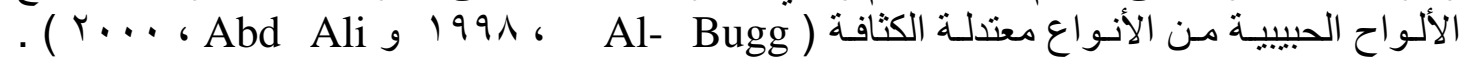

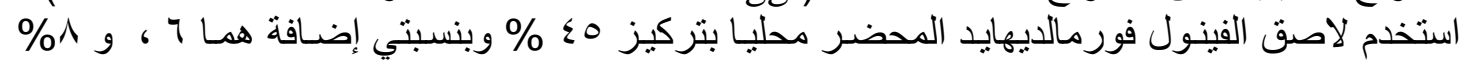

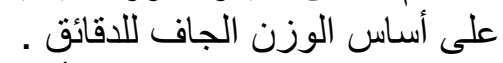
نفذ البحث بمرحلتين وكما يأتي:

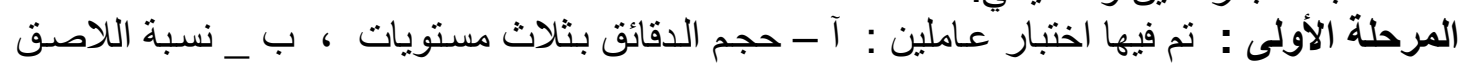

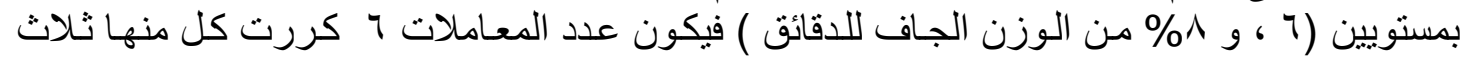

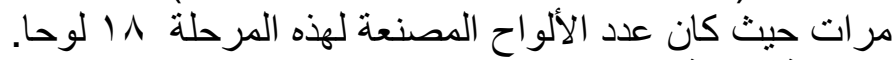

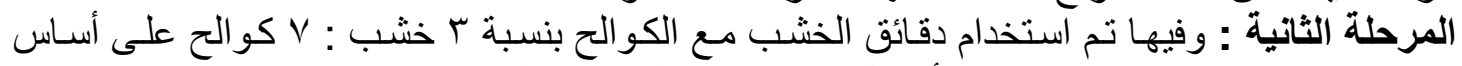

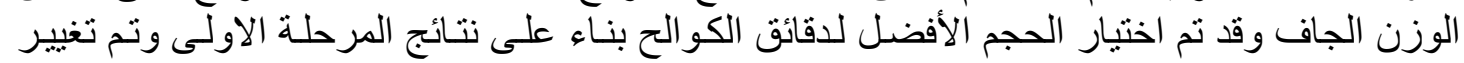

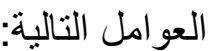

آ - نوع اللوح المنتج : بمستوبين همـا : الواح احاديـة الطبقة ، و ألو اح ثلاثيـة الطبقة بحيث شكل

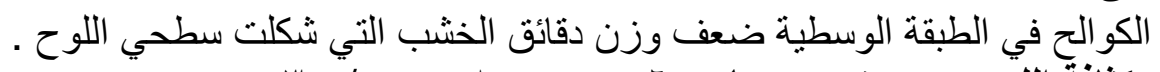

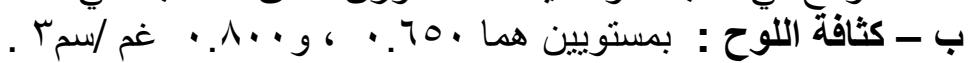

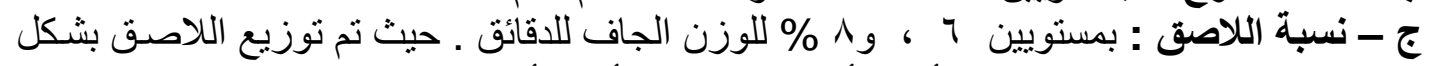

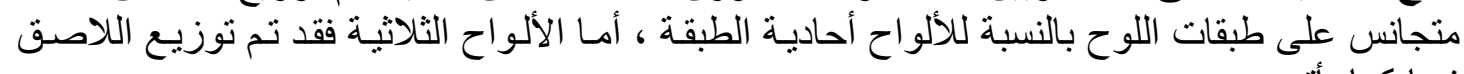

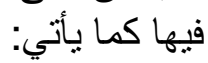

محتوى دقائق الوسط (\%)

محتوى دقائق السطح (\%)

المحنوى الكلّي للاصق (\%)

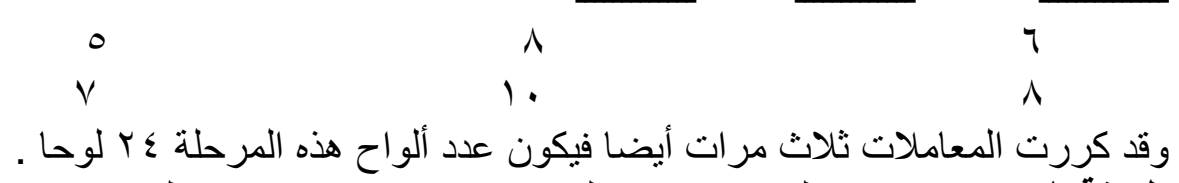

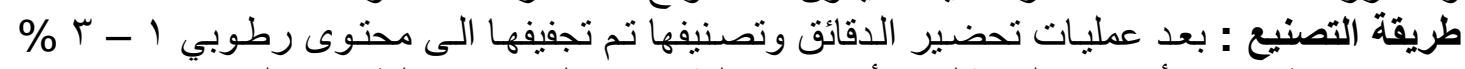

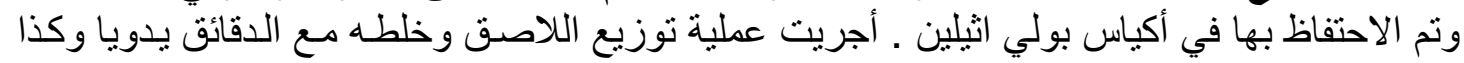
بالنسبة لعمليتي تكوين الحصير ( Mat Forming ) و و الكبس المسبق ( Prepressing ) ـ وقد تم تم أثناء التصنيع تثبيت العو امل التالية: 


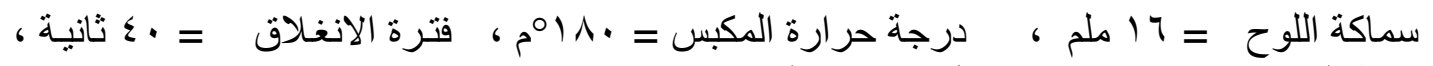

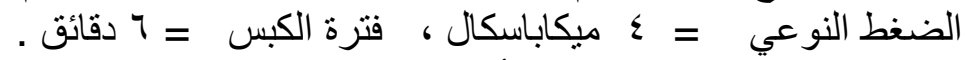

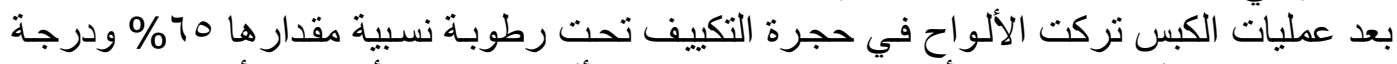

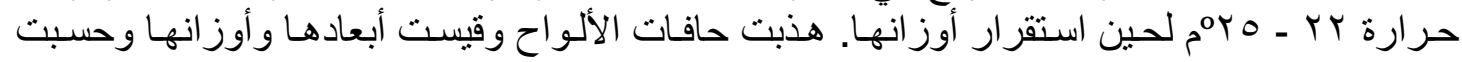

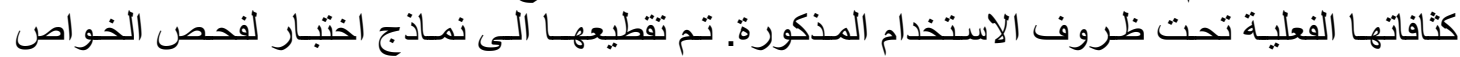

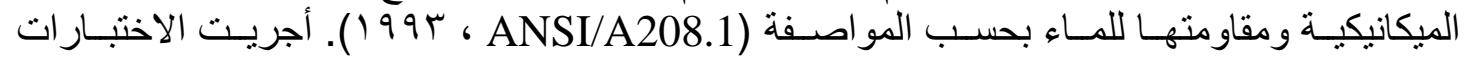

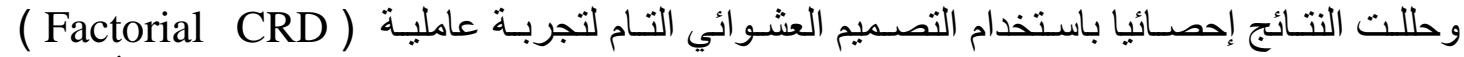
باعتبار التجربة الاولى ذات معاملتين بستة تداخلات و الثنانية ذات ثثلاث معاملات بستة تداخلات التات أيضا .

\section{النتائج والمناقشة التشة}

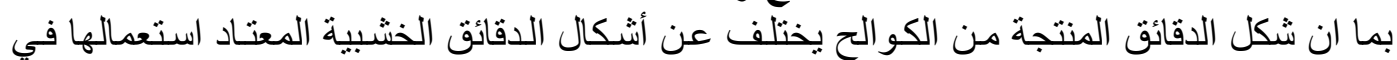

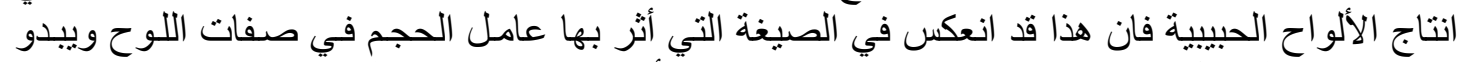

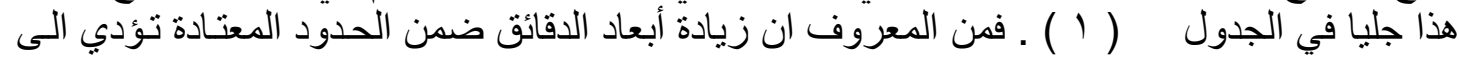

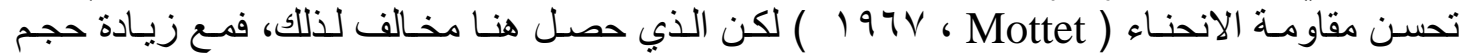

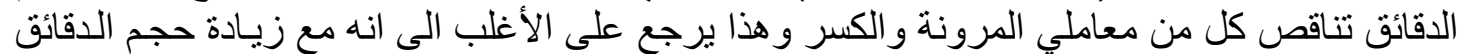

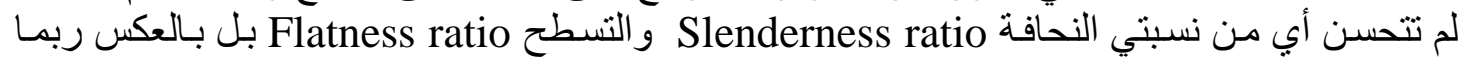

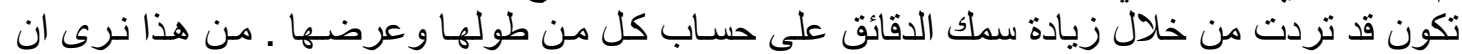

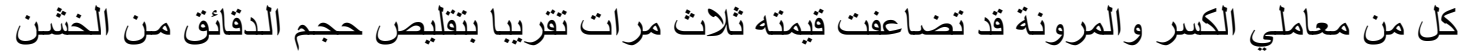

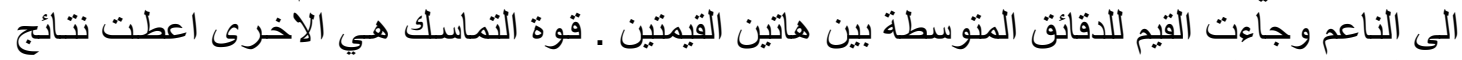

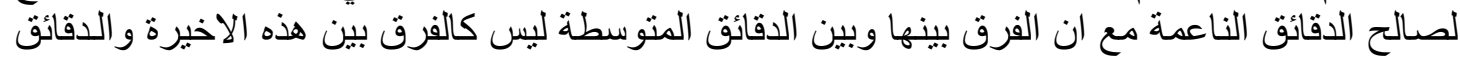

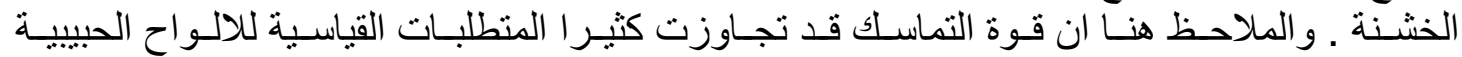

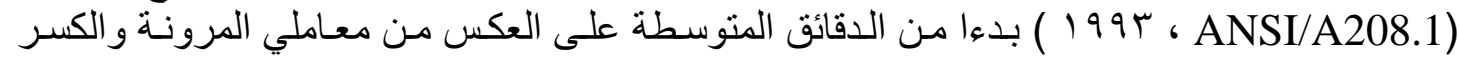

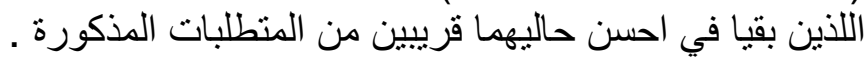

الجدول ( 1 ) : تأثثر حجم الدقائق وكمية اللاصق في صفات الالواح احادية الطبقة

\begin{tabular}{|c|c|c|c|c|c|c|c|c|c|}
\hline \multicolumn{3}{|c|}{ دقائق نـاعمــة } & \multicolumn{3}{|c|}{ دقائق متوسـطة } & \multicolumn{3}{|c|}{ دقائق خشــــة } & \multirow[t]{2}{*}{ صفــات اللوح ح } \\
\hline المعدل & $* \% \wedge$ & *\% & المعدل & $* \% \wedge$ & *\% & المعدل - ال & $* \% \wedge$ & *\% 1 & \\
\hline. $.70 \mathrm{~V}$ &. $.70 \mathrm{~V}$ & .701 & $.7 \leqslant 7$ & $\cdot .7 \leqslant 9$ & $. \quad T \leqslant r$ & $\cdot .7 \leqslant \wedge$ &. $.70 Y$ & $. .7 \leq \leqslant$ & كثافة اللوح (غد/سم؟) \\
\hline $\begin{array}{l}r \leq 99 \\
(ج)\end{array}$ & $r \leqslant 10$ & IAIT & $\begin{array}{l}9 \leqslant 1 \\
(4)\end{array}$ & $1 . V V$ & $\Lambda \cdot \varepsilon$ & $\begin{array}{l}V \Psi \varepsilon \\
(i)\end{array}$ & VVY & $79 r$ & معامل المرونة (Mpa) \\
\hline $\begin{array}{l}11.50 \\
(ج)\end{array}$ & TY.T & $1 \cdot . \mathrm{VV}$ & $\begin{array}{l}7 . r 4 \\
(4)\end{array}$ & $7.7 \mathrm{~V}$ & 0.10 & $\begin{array}{l}r .9 \\
(i)\end{array}$ & $T .9$ & 5.11 & معامل الكسر (Mpa) \\
\hline $\begin{array}{l}\cdot \because r \\
\text { (ج) }\end{array}$ & $\cdot V 7 \varepsilon$ & $\cdot .774$ & $\begin{array}{l}\because 0 \gamma \wedge \\
(ب)\end{array}$ & $\cdot . V \Sigma$. & $\because \varepsilon 17$ & $\begin{array}{l}\cdot Y^{K} \\
(1)\end{array}$ & .150 & $\cdot .1 \cdot 1$ & قوة التماسك (Mpa) \\
\hline $\begin{array}{l}0.9 \\
(\varphi)\end{array}$ & $\varepsilon 1 . \xi$ & 7.4 & $\begin{array}{l}\text { EV.T } \\
(\varphi)\end{array}$ & $\varepsilon 1 . V$ & 04.0 & $\begin{array}{l}11.79 \\
(i)\end{array}$ & $11 \cdot .7$ & ITr. & نسبة الماء الممتصة واحدة \\
\hline $\begin{array}{l}7 \varepsilon .1 \\
(ب)\end{array}$ & 0 or.1 & Vo.1 & $\begin{array}{l}47 \\
(4.7 \\
(4)\end{array}$ & 01.9 & 77.5 & $\begin{array}{l}110.7 \\
(i)\end{array}$ & $M \cdot \cdot r$ & $1 \pi 1.0$ & نسبة الماء الممتصة باعة \\
\hline $\begin{array}{l}\text { Ar.r } \\
(\varphi)\end{array}$ & VO.Y & 19.r & $\begin{array}{l}\text { NV.T } \\
(+)\end{array}$ & AT.\& & $91 . \mathrm{V}$ & $\begin{array}{l}149.0 \\
(1)^{\circ}\end{array}$ & $1 \pi{ }_{1} 1$ & $1 \leqslant 7.9$ & نسبة الماء المنصة \\
\hline $\begin{array}{l}14.9 \\
(4)\end{array}$ & $1 Y .0$ & 10.5 & $\begin{array}{l}10.1 \\
(4)\end{array}$ & IT.1 & $1 \Lambda$. & $\begin{array}{l}01.7 \\
(i)\end{array}$ & 0 or.1 & $\varepsilon 9 . \varepsilon$ & نسبة الانتفاخ احدة \\
\hline $\begin{array}{l}17.1 \\
(+)\end{array}$ & 17.0 & 17.1 & $\begin{array}{l}19.4 \\
(4)\end{array}$ & 17.7 & $r 1.9$ & $\begin{array}{l}00.1 \\
(i)\end{array}$ & $00 . r$ & 00.1 & نسبة الانتفاخ \\
\hline $\begin{array}{l}r r . q \\
(+)\end{array}$ & Y1.9 & rฯ. & $\begin{array}{l}r \varepsilon, Y \\
(ب)\end{array}$ & $r, Y$ & TY.Y & $\begin{array}{l}09.1 \\
(i)\end{array}$ & 07.1 & TY.V & نسبة الانتفاخ \\
\hline
\end{tabular}

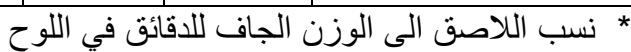

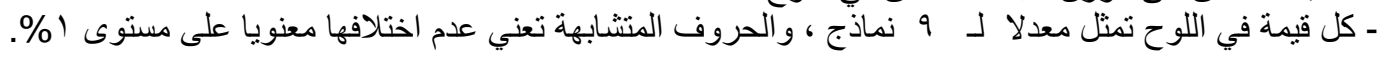

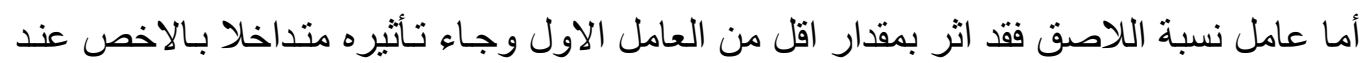

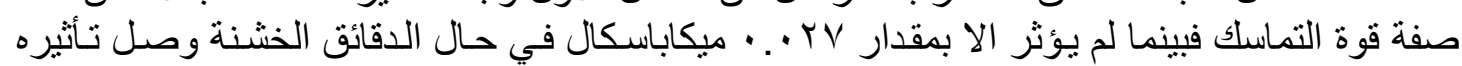




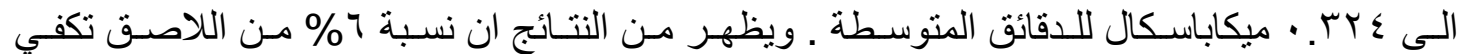

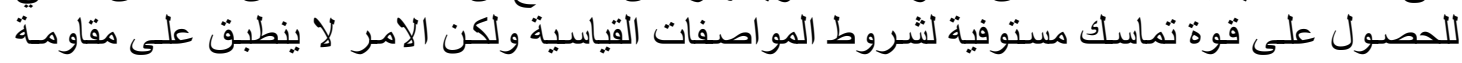
الانحناء.

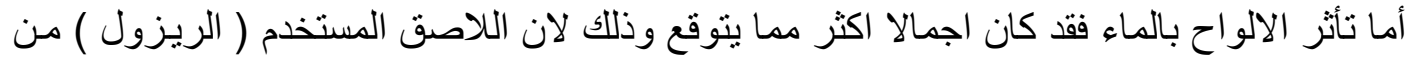

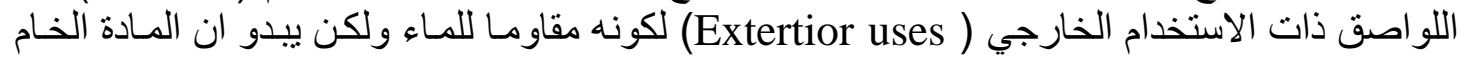

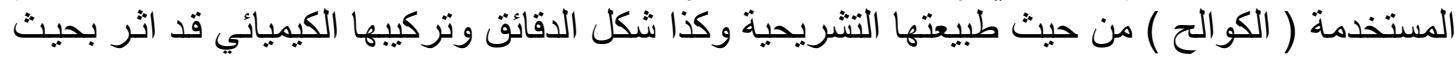

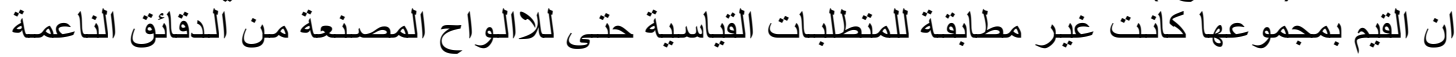

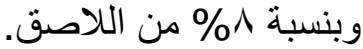

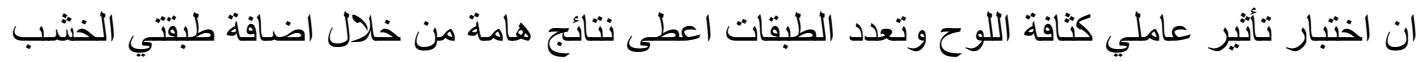

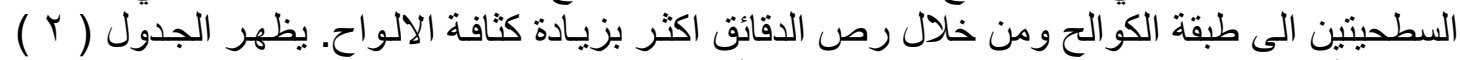

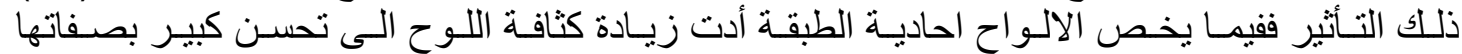

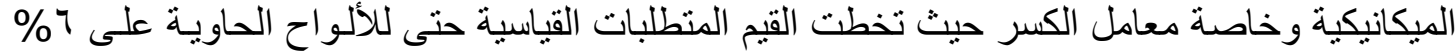

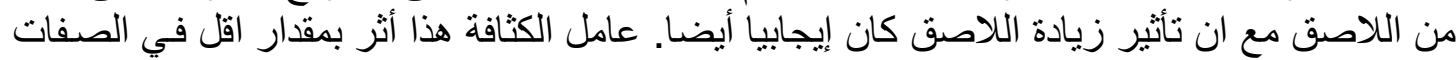

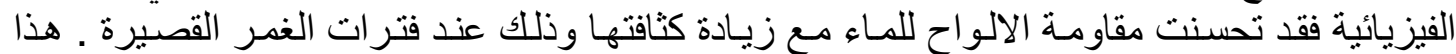

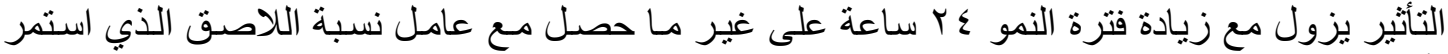
تأثثره مع طول فترة الغمر ذلك على الاغلب لكون الكون اللاصق المستخدم مقاوم للماء اصلا.

جدول ( r ) ): تأثثير تعدد الطبقات وكثافة اللوح ونسبة اللاصـق في صفات الالـواح الحبيييـة احاديـة

\begin{tabular}{|c|c|c|c|c|c|c|c|c|c|c|c|c|}
\hline \multicolumn{3}{|c|}{ ثلاثية الطبقة عالية } & \multicolumn{3}{|c|}{ ثلاثية الطبقة متوسطة } & \multicolumn{3}{|c|}{ احادية الطنقة عالية } & \multicolumn{3}{|c|}{ احادية الطبقة متوسطة الكثافة } & \multirow[t]{2}{*}{ اللوح } \\
\hline المعدل & $\% \wedge$ & $\%\urcorner$ & المعدل & $\% \wedge$ & $\% 4$ & المعدل & $\% \wedge$ & $\% 7$ & المعدل & $\% \wedge$ & $\% 4$ & \\
\hline$\because \vee 91$ & $\cdot \vee \wedge \wedge$ & $\cdot . \vee 9 \leq$ & $.70 \mathrm{r}$ & $.7 \leqslant 9$ & $\cdot .70 \leqslant$ & $\because$ VAT & .991 & . VAr & $. .70 \leqslant$ & $.70 \mathrm{~V}$ &. .701 & كثافة اللوح \\
\hline $\begin{array}{l}r \leqslant \mu \wedge \\
(ج)\end{array}$ & r9o. & raY4 & $\begin{array}{l}\text { roog } \\
(ب)\end{array}$ & TV.T & $r \leqslant 17$ & $\begin{array}{l}(ب) 7 \\
(ب)\end{array}$ & TQYY & YT.q & $\begin{array}{l}r) \leqslant 9 \\
(1)\end{array}$ & $r \leqslant 10$ & 1114 & $\begin{array}{r}\text { المرونة } \\
\text { (Mpa) }\end{array}$ \\
\hline $\begin{array}{l}r \leq . V A \\
(د)\end{array}$ & ro. T & $r \leq . r q$ & $\begin{array}{l}1 \leq . r \cdot \\
(\varphi)\end{array}$ & $1 \leq .14$ & $1 \leq . Y 4$ & $\begin{array}{l}17.9 . \\
(ج)\end{array}$ & $1 v .0$. & $17 . r$. & $\begin{array}{l}11.10 \\
(i)\end{array}$ & IT.M & $1 \cdot . V V$ & $\begin{array}{l}\text { الكسر } \\
\text { (Mpa) }\end{array}$ \\
\hline $\begin{array}{l}\cdot \because \text { VAV } \\
(ج)\end{array}$ & $\therefore .109$ & \& & $\begin{array}{c}.70 \mathrm{~V} \\
(1)\end{array}$ & $\because Y Y$. & .091 & $\begin{array}{l}\because A r \varepsilon \\
(ج)\end{array}$ & .949 & $\cdot v \cdot \wedge$ & $\begin{array}{l}\cdot{ }^{v}(r) \\
(ب)\end{array}$ & 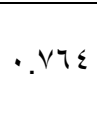 & אדית. & قوة التماسك \\
\hline $\begin{array}{l}\text { r). } \\
(1)^{9}\end{array}$ & IV. & r..A & $\begin{array}{l}0.11 \\
(ج)\end{array}$ & $\leqslant \Lambda_{.}$. & or.t & $\begin{array}{l}r Y . q \\
(ب)\end{array}$ & 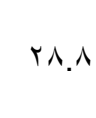 & $\varepsilon V_{.}$. & $\begin{array}{l}0.99 \\
(ج)\end{array}$ & $\varepsilon 1 . \varepsilon$ & $\pi, r$ & $\begin{array}{c}\text { الامتصاص) } \\
\text { ساعة واحدة }\end{array}$ \\
\hline $\begin{array}{l}\text { res } \\
(i)^{2}\end{array}$ & YA.r & $r \leqslant .0$ & $\begin{array}{l}(1.0 \\
(2)\end{array}$ & $v \cdot .1$ & VT. 9 & $\begin{array}{l}\text { or. } \\
(ب)\end{array}$ & ro.r & 79.7 & $\begin{array}{l}7 \leqslant .1 \\
(\text { ) }\end{array}$ & or. & vo.1 & 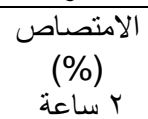 \\
\hline $\begin{array}{l}\text { Ty.A } \\
(i)\end{array}$ & 07.9 & $V \Lambda . V$ & $\begin{array}{l}94.9 \\
\text { (ج) }\end{array}$ & $19 . \wedge$ & 97. & $\begin{array}{l}V r \cdot \hat{A} \\
(\varphi)\end{array}$ & $v \cdot . \varepsilon$ & VY. & (ب ) & OY.r & 19. & 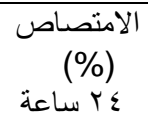 \\
\hline$\left(\begin{array}{l}A \\
(i)\end{array}\right.$ & 0.9 & $1 . \mu$ & (ب) & $11 . \mathrm{V}$ & 17.4 & $\begin{array}{l}1 Y .9 \\
(ب)\end{array}$ & 11.1 & $1 \leq . \wedge$ & (ب) & IT.r & 10.0 & 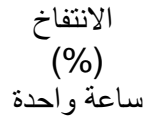 \\
\hline $\begin{array}{l}i r .1 \\
(i)\end{array}$ & V.r & 17.9 & $\begin{array}{l}19.0 \\
(ج)\end{array}$ & $17 . \mathrm{V}$ & KY.Y & $\begin{array}{l}10.1 \\
(ب)\end{array}$ & $1 \leqslant . \mu$ & IV.r & $\begin{array}{l}17.8 \\
(ب)\end{array}$ & 17.0 & 17.1 & $\begin{array}{l}\text { الانتفاخ) } \\
\text { باعة } \\
\text { باعة }\end{array}$ \\
\hline $\begin{array}{l}r r . \\
(i)\end{array}$ & IV.A & YA. Y & $\begin{array}{l}\text { ro.r } \\
\text { (ج) }\end{array}$ & r). 9 & $r \Lambda . \varepsilon$ & (ب). & rY.A & ro.r & $\begin{array}{l}r r . q \\
\text { (ب) }\end{array}$ & r). 9 & r.. & 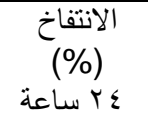 \\
\hline
\end{tabular}




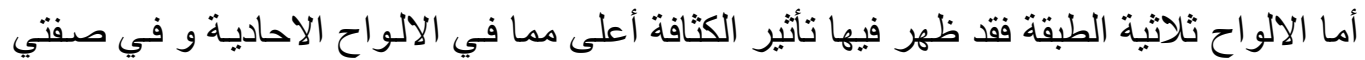

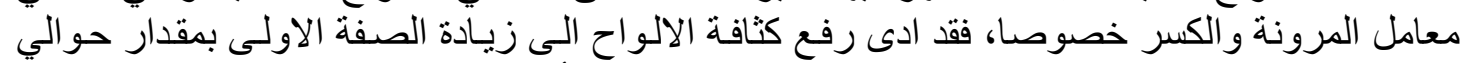

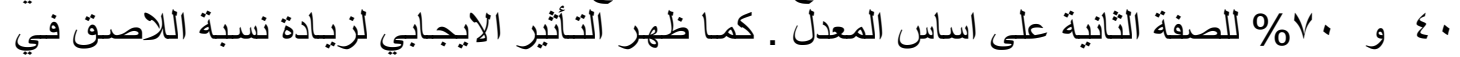

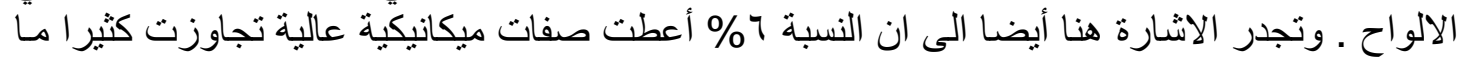

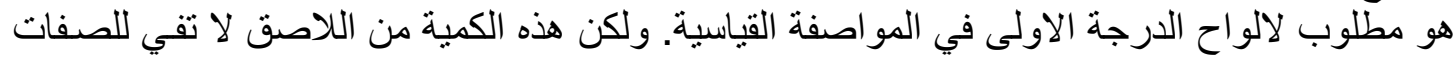
الفيزيائية وبالاخص لالواح للالو اح متوسطة الاولى فئافة.

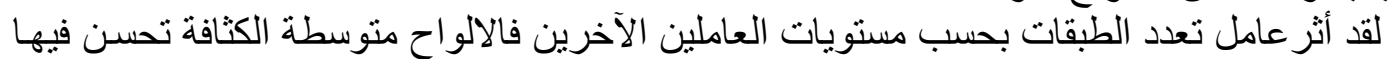

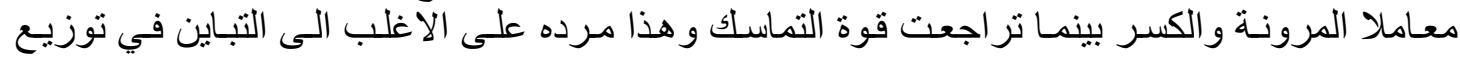

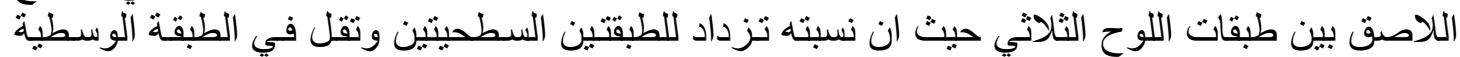

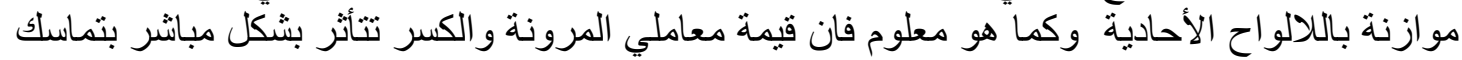

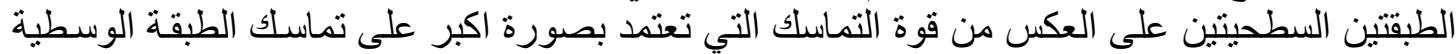

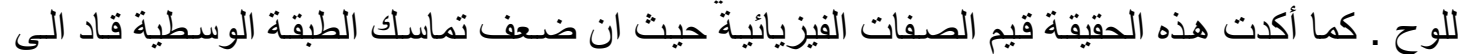

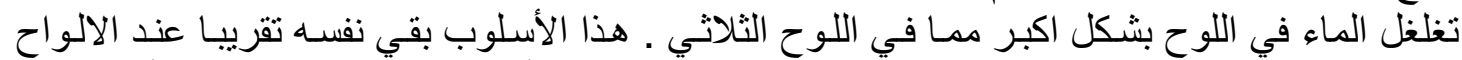

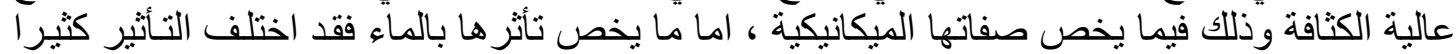

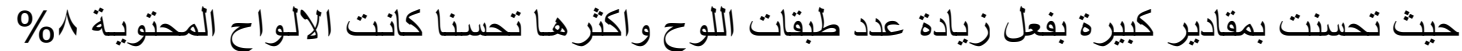

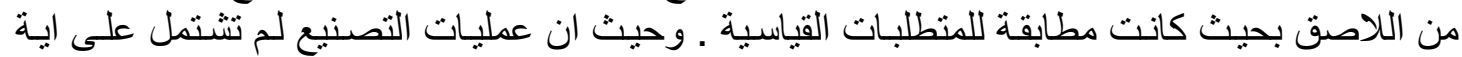

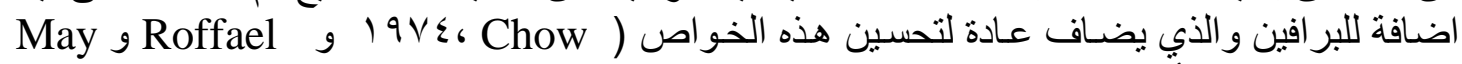

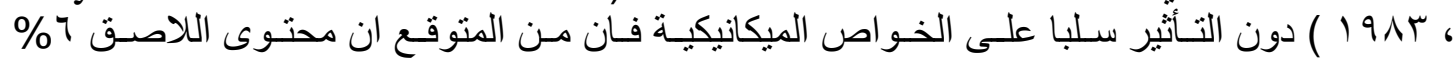
سيعطي قيما مستوفية للمتطلبات في حال استخدام البر افين لا سيما وان صفاته كانت قريبة جدا من ذلك الك

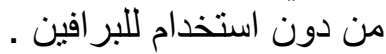

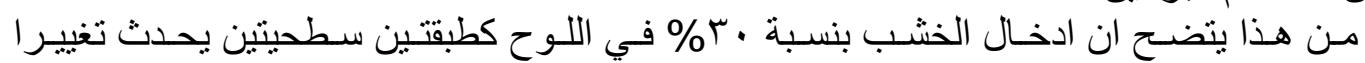

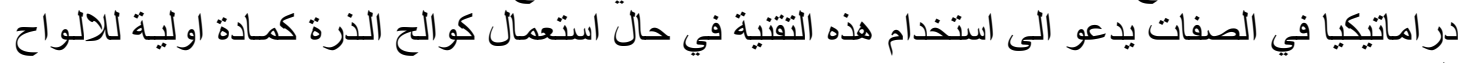
الحيبية

\title{
UTILIZATION OF CORN COBS IN THE MANUFACTURING OF PARTICLEBOARDS
}

Basim A. Abd Ali

Natural Hist. Research Cent. \& Museum

Baghdad Univ. Iraq
Nazar K. Al- dainy

Construction Research Center

Ministry of Industry

\begin{abstract}
The research has conducted to investigate the possibility of corn cobs utilization in particleboards production. Forty two boards were manufactured within two experiments. At the first one 18 boards produced to test the effect of two factors ; particle size ( 3 levels ), and resin content ( 2 levels ) on the board properties. The results of this experiment affirmed the superiority of the smallest size of particles. The second one dealt with the influence of three factors ; board layering, resin content, and board density. Half of 24 boards of this stage were manufactured by using wood particles as board surfaces to the corn cobs core. Testing results showed that 3 - layer board were more better than single layer one in all of mechanical and physical properties. This difference was at its higher level in case of high density boards. In spite of the significant improvement occurred with increasing resin content from $6 \%$ to $8 \%$ the first one seemed to be sufficient especially with 3 layers high density boards. Hence, the introducing of wood in a $3: 7$ ratio with corn cobs resulted
\end{abstract}


in a very high improvement that should not neglected when trying in the utilization of this raw material.

\section{(المصادر}

Abd Ali , B. A. , and W. A. Kasir (1997). Production of particleboard from sun flower stalks. Mesopotamia J. of Agric. 29 (3) : 75 - 81 .

Abd Ali , B.A. ( 2000 ). Effect of some processing variables on the properties of pine particleboards. Mesopotamia J. of Agric., 32 (3) : 80-85.

Abd Ali, B. A. (2003). Study on the suitability of Suaeda fruticosa as raw material for particleboards. Mesopotamia J. of Agric., 34 (3) : 62 67 .

Al - Bugg , Y . S . ( 1998 ). Deduction of typical press cycle in manufacturing particleboardsfrom poplar. Unpnblished Ph . D . thesis, Mosul University . $79 \mathrm{pp}$.

Al - Sudani, O. , D. Daou and S. Micahael (1988). Properties of p[articleboards from reed - typha mixtures . J. Pet. Res., 7 (1) : 197 208 .

ANSI / A208.1 (1993). Amereican national standards. 18928 Premiere Court Gaithersburg. MD 20879 - 1569, USA .

Cao, N. , M. Krishman, J. Du , C. gong , N. ho , Z. Chen and G. Tsao (1996). Ethanol Production from corn cob pretreated by the ammonia steeping process using genetically engineered yeast . Biotechnology letter : $1013-1018$.

Chow P. (1974). Dry - formed composite boards from selected agricultural residues . World consultation on Wood - based panels . FAO, New Delhi, India.

Jingxing , C. (1988). Review of the bagasse particleboard production technology in China. UNIDO Rept. UNIDO - ID/WG . 476 / 6 , 16pp.

Marri , S. , L. Tabil , and A. Opuku (2002). Ethanol from agricultural crop residues - an overview paper No. MBSK 02 - 217 An ASAI / CSAE Meeting presentation, 23pp.

Mottet, L. M. (1967). The particle geometry factor in particleboard manufacturing. Proc. $1^{\text {st }}$ Int. Particleboard Symp., Washington State Univ. , Pullman , 23 - 73 .

Roffael , E. and H. May (1983). Paraffin sizing of particleboards ; chemical asoects . Proc. $17^{\text {th }}$ Int. Particleboard Symp., Washington State Univ., Pullman , 283 - 295.

Rowell, R. M. (1995). Composite materials from agricultural resources. Research in industrial application of non food crops I : Plant fibers ; Proc. Of Seminar ; Denmark Academy of Technical Science : 27 - 41 .

Troger , F. , and G. Pinke (1988). Manufacture of boards glued with polymeric diphenylmethane $-4-4$ diisocyanate containing varios proportions of straw . Holz als Roh Werkstoff, 46 (10) : $389-395$.

UNIDO (1975). Review of agricultural residues utilization for production of panels . FO / WCWBP / 75 Doc. 75 . 
Vlades, J. , J. Mendes , M. rodreguez and P. Sosa (1989). Quality of bagasse particleboards in Cuba . Revista ICIDCA Sobre Los Derivados de la Cana de Azucar , 23 (1) : $44-47$.

Youngquist J., B. English, H. Spelter and P. Chow (1993). Agricultural fibers in composition panels . In. Proc. 27 ${ }^{\text {th }}$ Int. Particleboard, Composite materials Symp. Washington State Univ., Pullman, 133 152 .

Youngquist J., A. Krzysik , B. English, H. Spelter , and P. Chow (1996).Agricultural fibers for use in building components. WI : Forest product Society Proc., $7286: 123-134$. 